\title{
Infinite Number of Disjoint Chaotic Subsystems of Cellular Automaton Rule 106
}

\author{
Gaocang Zhao1, Fangyue Chen1, Weifeng Jin² \\ ${ }^{1}$ School of Science, Hangzhou Dianzi University, Hangzhou, China \\ ${ }^{2}$ College of Pharmaceutical Sciences, Zhejiang Chinese Medical University, Hangzhou, China \\ Email: gaoluncangcang@qq.com, fychen@hdu.edu.cn, iin.weifeng@hotmail.com
}

Received 24 August 2014; revised 20 September 2014; accepted 8 October 2014

Copyright (C) 2014 by authors and Scientific Research Publishing Inc.

This work is licensed under the Creative Commons Attribution International License (CC BY). http://creativecommons.org/licenses/by/4.0/

\begin{abstract}
In this paper, the dynamics of rule 106, a Chua's hyper Bernoulli cellular automata rule, is studied and discussed from the viewpoint of symbolic dynamics. It is presented that rule 106 defines a chaotic subsystem which is topologically mixing and possesses the positive topologically entropy. An effective method of constructing its chaotic subsystems is proposed. Indeed, it is interesting to find that this rule is filled with infinitely many disjoint chaotic subsystems. Special attention is paid to each subsystem on which rule 106 is topologically mixing and possesses the positive topologically entropy. Therefore, it is natural to argue that the intrinsic complexity of rule 106 is high from this viewpoint.
\end{abstract}

\section{Keywords}

Cellular Automata, Chaos, Topologically Entropy, Topologically Mixing, Subsystem

\section{Introduction}

Cellular Automata (CA), first conceived around 1950 by von Neumann [1], are a class of spatially and temporally discrete mathematical structure by local interactions and an inherently parallel form of evolution. The whole structure is able to produce complex and interesting dynamical phenomena by means of designing simple transition rule. Due to their simple mathematical constructions and distinguishing features, CA have drawn a great deal of attention from various scientists. In 1969, the study of topological dynamics of CA was developed by Hedlund [2], who viewed one-dimensional CA in the context of symbolic dynamics as endomorphisms of the shift dynamical system, where the main results are the characterizations of surjective and open CA. In 1970, Conway proposed game of life [3], which received widespread interests among researchers in different fields. In the early 1980s, Wolfram proposed CA as models for physical systems exhibiting complex or 
even chaos behaviors and elementary CA (ECA) that consist of a one-dimensional array of finite binary cells, each interacting only with the two nearest neighbors [4]-[6]. He classified 256 ECA rules informally into four classes using dynamical concepts like periodicity, stability and chaos. In 2002, Wolfram introduced his work A New Kind of Science [6]. Based on this work, Chua et al. have concluded the dynamics of ECA from a nonlinear dynamics perspective [7]-[10]. And he divided 256 ECA rules into four classes: period- $k$ rules $(k=1,2,3)$, Bernoulli-shift rules, complex Bernoulli-shift rules and hyper Bernoulli-shift rules.

Gratefully, the research of CA has drawn more and more scientists' attention in the last 20 years. Many concepts of topological dynamics have been used to describe and classify them [11]-[15]. And the dynamical properties of some robust Bernoulli-shift rules have been studied in the bi-infinite symbolic sequence space [14], [15]. Rule 106 belonging to hyper Bernoulli-shift rules possesses complex and distinctive dynamical behaviors. In a paper [16], the authors introduced the notion of permutivity of a map in a certain variable. Then they proved that every one-dimensional CA based on the local rule which is permutive either in the leftmost or rightmost variable is Devaney chaotic. Rule 106 is in this situation. Presently, this work is devoted to an in-depth study of rule 106 from the perspective of nonlinear dynamics under the framework of bi-infinite symbolic sequence space, and mainly studies the complex dynamics on its infinite number of subsystems.

The rest of the paper is organized as follows: Section 2 presents the basic concepts of one-dimensional CA and symbolic dynamics. Based on these concepts, it shows a subsystem of rule 106. Section 3 explores the complex dynamical behaviors of rule 106. Section 4 describes that there exist infinitely many disjoint chaotic subsystems in this chaotic subsystem. Finally, Section 5 concludes this paper.

\section{Preliminaries}

For a finite symbol $S$, a word over $S$ is finite sequence $a=\left(a_{0}, a_{1}, \cdots, a_{n-1}\right)$ of elements of $S$. The length of a is denoted by $|a|=n$. Denote the set of all words of length $n$ by $S^{n}$. If $a$ is a finite or infinite word and $I=[i, j]$ is an interval of integers on which $a$ is defined, put $a_{[i, j]}=\left(a_{i}, \cdots, a_{j}\right)$ and $a_{[i, j]}=\left(a_{i}, \cdots, a_{j-1}\right) . b$ is a subword of $a$, denoted by $b \prec a$, if $b=a_{I}$, for some interval $I \subseteq Z$; otherwise, denoted $b \nprec a$. The set of bi-infinite configurations is denoted by $S^{Z}$ and a metric " $d$ ” on $S^{Z}$ is defined as $d(x, \bar{x})=\sum_{i=-\infty}^{\infty} \frac{1}{2^{|i|}} \cdot \frac{\bar{d}\left(x_{i}, \bar{x}_{i}\right)}{1+\bar{d}\left(x_{i}, \bar{x}_{i}\right)}$, where $x, \bar{x} \in S^{z}$, and $\bar{d}(\cdot, \cdot)$ is the metric on $S$ defined as $\bar{d}\left(x_{i}, \bar{x}_{i}\right)=\left\{\begin{array}{ll}1, & \text { if } x_{i}=\bar{x}_{i} \\ 0, & \text { if } x_{i} \neq \bar{x}_{i}\end{array}\right.$. It is well known that $S^{Z}$ is a compact, perfect and totally disconnected metric space.

By a theorem of Hedlund, a map $T: S^{Z} \rightarrow S^{Z}$ is a cellular automata iff it is continuous and commutes with shift map $\sigma$, i.e., $T \circ \sigma=\sigma \circ T$, where $\sigma: S^{Z} \rightarrow S^{Z}$ is defined by $[\sigma(x)]_{i}=x_{i+1}$. For any CA there exists radius $r \geq 0$ and a loca rule $N: S^{2 r+1} \rightarrow S$ such that $[T(x)]_{i}=N\left(x_{[i-r, i+r]}\right)$. Moreover, $\left(S^{Z}, T\right)$ is a compact dynamical system. To enhance readability, it is desirable to write a CA as $T_{N}$ for local rule $N$.

A set $X \subseteq S^{Z}$ is $T$-invariant if $T(X) \subseteq X$, and strongly $T$-invariant if $T(X)=X$. If $X$ is closed and $T$-invariant, then $(X, T)$ or simply $X$ is called a subsystem of $T$. For instance, let $\mathscr{C}$ denote a set of some finite words over $S$, and $\Lambda_{\mathscr{C}}$ is the set which consists of the bi-infinite configurations made up of all the words in $\mathscr{A}$. Then $\Lambda_{\mathscr{C}}$ is subsystem of $\sigma$, where $\mathscr{A}$ is said to be the determinative block system of $\Lambda_{,}$.

For bi-infinite ECA, $r=1$ and $S$ is denoted by $\{0,1\}$. Each local rule can be expressed by a Boolean function. For example, the Boolean function of rule 106 is $N\left(x_{[i-1, i+1]}\right)=x_{i-1} \cdot x_{i} \cdot \bar{x}_{i+1} \oplus \bar{x}_{i} \cdot x_{i+1} \oplus \bar{x}_{i-1} \cdot x_{i} \cdot x_{i+1}$, $\forall i \in Z$, where “.”, “ $\oplus$ ” and “-” stand for “AND”, “XOR” and "NOT” logical operations, respectively [11]. Thus the global map of rule 106 is induced as follows: for any $x \in S^{Z}$, 
$\left[T_{106}(x)\right]_{i}=x_{i-1} \cdot x_{i} \cdot \bar{x}_{i+1} \oplus \bar{x}_{i} \cdot x_{i+1} \oplus \bar{x}_{i-1} \cdot x_{i} \cdot x_{i+1}, \quad \forall i \in Z$, where $\left[T_{106}(x)\right]_{i}$ denotes the $i$ th symbol of $T_{106}(x)$. For clarity, the truth table of rule 106 is depicted in Table 1.

Based exclusively on this truth table, a subsystem of rule 106 in $S^{Z}$ is shown as follows.

Proposition 1. For rule 106, there exists a subset $\Lambda \subset S^{Z}$ such that $\left.T_{106}\right|_{\Lambda}=\left.\sigma\right|_{\Lambda}$ iff $\forall x \in \Lambda$, $x_{[i-1, i+1]} \in \mathscr{C}, \forall i \in Z$, where $\mathscr{C}=\{(0,0,0),(0,0,1),(0,1,0),(1,0,0),(1,0,1)\}$.

Proof: (Necessity) Suppose that there exists a subset $\Lambda \subset S^{Z}$ such that $\left.T_{106}\right|_{\Lambda}=\left.\sigma\right|_{\Lambda}$, then, $\forall x=\left(\cdots, x_{-1}, x_{0}, x_{1}, \cdots\right) \in \Lambda$, one has $\left[T_{106}(x)\right]_{i}=[\sigma(x)]_{i}=x_{i+1}, \forall i \in Z$ According to the Boolean function of rule 106, one has $x_{i-1} \cdot x_{i} \cdot \bar{x}_{i+1} \oplus \bar{x}_{i} \cdot x_{i+1} \oplus \bar{x}_{i-1} \cdot x_{i} \cdot x_{i+1}=x_{i+1}$ this implies $x_{i-1} \cdot x_{i}=0$ so $x_{i-1}$ and $x_{i}$ can not be 1 simultaneously, $\forall i \in Z$. Additionally, if there exists $i_{0}$ such that $\left(x_{i_{0}-1}, x_{i_{0}}, x_{i_{0}+1}\right)=(0,1,1)$ then it must satisfy that $\left(x_{i_{0}}, x_{i_{0}+1}, x_{i_{0}+2}\right)=(1,1,0)$ or $(1,1,1)$, this is contradictory with $x_{i_{0}} \cdot x_{i_{0}+1}=0$ Hence, the determinative block system of $\Lambda$ is $\mathscr{C}=\{(0,0,0),(0,0,1),(0,1,0),(1,0,0),(1,0,1)\}$.

(Sufficiency) The proof of sufficiency can be verified directly, the details are omitted here. The proof of the proposition is completed.

For illustration, simulations of the spatial and temporal evolution of rule 106 with a random initial configuration and an initial configuration of $\Lambda$ are shown in Figure 1, where the black pixel stands for 1 and white for 0 .

\section{Complex Dynamics of $T_{106}$}

In this section, the dynamical behaviors of $T_{106}$ on $\Lambda$ are exploited. As the topological dynamics of a subshift of finite type is largely determined by the properties of its transition matrix, it is helpful to briefly review some definitions from [17]. A matrix A is positive if all of its entries are nonnegative; irreducible if $\forall i, j, \exists n$ such that $A_{i, j}^{n}>0$; aperiodic if $\exists N$, such that $A_{i, j}^{n}>0, n>N, \forall i, j$. If $\Lambda_{A}$ is a 2-order subshift of finite type,

\section{Table 1. Logical table of rule 106.}

\begin{tabular}{cccc}
\hline$\left(x_{i-1}, x_{i}, x_{i+1}\right)$ & {$\left[T_{106}(x)\right]_{i}$} & $\left(x_{i-1}, x_{i}, x_{i+1}\right)$ & {$\left[T_{106}(x)\right]_{i}$} \\
\hline 000 & 0 & 100 & 0 \\
001 & 1 & 101 & 1 \\
010 & 0 & 110 & 1 \\
011 & 1 & 111 & 0
\end{tabular}

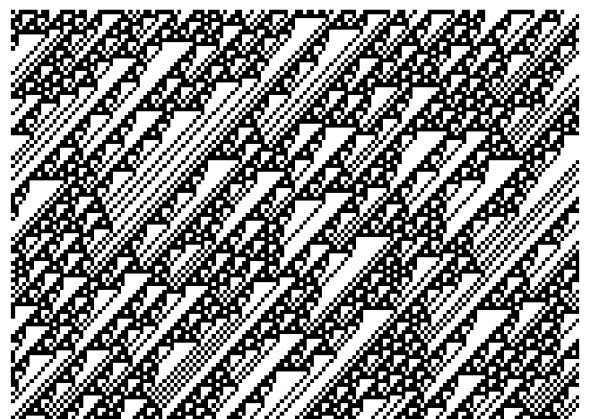

(a)

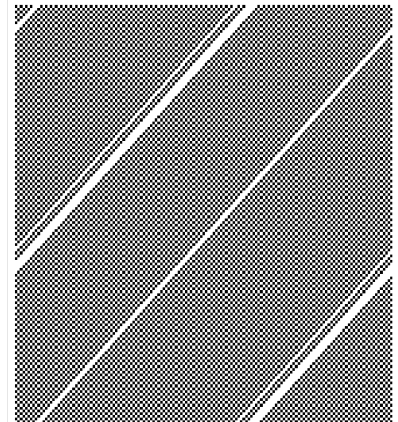

(b)

Figure 1. (a) The evolution of rule 106 from random initial configuration, (b) The evolution of rule 106 from an initial configuration of $\Lambda$. 
then the associated transition matrix $A$ is the $N \times N$ matrix with $A_{i, j}=1$, if $(i, j) \in \Lambda_{A}$; otherwise $A_{i, j}=0$. Denote a 2-order subshift of finite type by $\sigma_{A}: \Lambda_{A} \rightarrow \Lambda_{A}$ It is known that a 2-order subshift of finite type is topologically mixing if and only if its transition matrix is irreducible and aperiodic [17] [18].

The nonlinear dynamical behavior of $T_{106}$ on $\Lambda$ is discussed by establishing the topologically conjugate relationship between $(\Lambda, \sigma)$ and a 2-order subshift of finite type. Let $\hat{S}=\left\{r_{0}, r_{1}, r_{2}, r_{3}, r_{4}\right\}$ be a new symbolic set, where $r_{i}, i=0,1,2,3,4$, represent the elements in $\mathscr{A}$, respectively. Then one can construct a new symbolic space $\hat{S}^{Z}$ on $\hat{S}$. Denote by $\mathscr{P}=\left\{\left(r r^{\prime}\right) \mid r=\left(b_{0}, b_{1}, b_{2}\right), r^{\prime}=\left(b_{0}^{\prime}, b_{1}^{\prime}, b_{2}^{\prime}\right) \in \hat{S}\right.$, and $\left.b_{1}=b_{0}^{\prime}, b_{2}=b_{1}^{\prime}\right\}$. Then, the 2-order subshift $\Lambda_{0}$ of $\sigma$ is defined by $\Lambda_{\supset}=\left\{r=\left.\left(\cdots, r_{-1}, r_{0}^{*}, r_{1}, \cdots\right) \in \hat{S}^{Z}\right|_{r_{i}} \in \hat{S},\left(r_{i}, r_{i+1}\right) \prec \mathscr{Y}, \forall i \in Z\right\}$. Moreover, it is clear to see that the transition matrix A of the subshift $\Lambda_{O}$ is:

$$
A=\left(\begin{array}{lllll}
1 & 0 & 0 & 1 & 0 \\
0 & 0 & 0 & 1 & 0 \\
0 & 1 & 0 & 0 & 1 \\
0 & 0 & 1 & 0 & 0 \\
0 & 0 & 1 & 0 & 0
\end{array}\right) .
$$

Theorem 1. 1) $(\Lambda, \sigma)$ and $\left(\Lambda_{,}, \sigma\right)$ are topologically conjugate;

2) $\left.T_{106}\right|_{\Lambda}$ is topologically mixing;

3) the topological entropy of $\left.T_{106}\right|_{\Lambda}$ satisfies ent $\left(\left.T_{106}\right|_{\Lambda}\right)=\log (\rho(A))>0$, where $\rho(A)$ is the spectral radius of the transition matrix $A$.

Proof: 1) Define a map from $\Lambda$ to $\Lambda_{\Omega}$ as follows:

$$
\begin{aligned}
\pi: \Lambda & \rightarrow \Lambda \\
x=\left(\cdots, x_{-1}, x_{0}, x_{1}, \cdots\right) & \mapsto\left(\cdots, r_{-1}, r_{0}, r_{1}, \cdots\right)
\end{aligned}
$$

where $r_{i}=x_{[i-1, i+1]}, \forall i \in Z$. Then, it follows from the definition of $\Lambda_{\triangle}$ that for any $x \in \Lambda_{\triangle}$, one has $\pi(x) \in \Lambda_{2}$; namely, $\pi(\Lambda) \subseteq \Lambda$. Then, it is easily to check that $\pi$ is a homeomorphism and $\pi \circ \sigma=\sigma \circ \pi$. Hence, $(\Lambda, \sigma)$ and $(\Lambda, \sigma)$ are topologically conjugate.

2) A satisfies $A^{n}>0, \forall n \geq 4$; namely, $A$ is irreducible and aperiodic, which implies that $\sigma$ is topologically mixing on $\Lambda$. Then, one can deduce $\left.T_{106}\right|_{\Lambda}$ is topologically mixing according to Theorem 11 ) and Proposition 1.

3) As ent $(\sigma)=\log (\rho(A))=\log \left(\lambda_{0}\right)$, where $\rho(A)$ is the spectral radius of the transition matrix $A$ and $\lambda_{0}$ is the positive real root of $\lambda^{2}-\lambda-1=0$. And $(\Lambda, \sigma)$ and $\left(\Lambda_{,}, \sigma\right)$ are topologically conjugate, so $\operatorname{ent}\left(\left.T_{106}\right|_{\Lambda}\right)=\operatorname{ent}(\sigma)=\log (\rho(A))>0$.

It is noted that a positive topological entropy is an important signature of the complexity of the system. It follows from [18] that the positive topological entropy implies chaos in the sense of Li-Yorke. And the topologically mixing is a very complex property of dynamical systems. A system with topologically mixing property has many chaotic properties in different senses. Therefore, the above mathematical analysis provides the following result.

Theorem 2. 1) $T_{106}$ is chaotic in the sense of Li-Yorke;

2) $T_{106}$ is chaotic in the sense of both Li-Yorke and Devaney on $\Lambda$. 


\section{Infinitely Many Chaotic Subsystems of $T_{106}$ in $\Lambda$}

It is helpful to review some definitions and basic properties of releasing transformation before we discuss the dynamics of $T_{106}$ on infinite number of subsystems. Let $\tilde{S}=\{\tilde{a}, \tilde{b}\}$ be a symbolic set, where $\tilde{a}=\left(a_{0}, a_{1}, \cdots, a_{n-1}\right)$ and $\tilde{b}=\left(b_{0}, b_{1}, \cdots, b_{n-1}\right)$ represent new symbols, respectively, and $a_{i}, b_{i} \in\{0,1\}, i=0,1,2, \cdots, n-1$. Denote by $\tilde{S}^{Z}$ the space of bi-infinite configurations over $\tilde{S}$ and induce a matric “ $d$ ” onto $\tilde{S}^{Z}$ as defined in the preceding section. Then, the releasing transformation $R$ is defined as follows:

$$
\begin{aligned}
R: \tilde{S}^{Z} & \rightarrow S^{Z} \\
y & \mapsto z
\end{aligned}
$$

where

$$
Z_{i}=[R(y)]_{i}=\left\{\begin{array}{cc}
a_{0}, & \text { if } i \bmod n=0, \text { and } y_{\frac{i}{n}}=\tilde{a} ; \\
a_{1}, & \text { if }(i+1) \bmod n=0, \text { and } \frac{y_{\frac{i+1}{n}}=\tilde{a} ;}{a_{n},} \quad \text { if }(i+n-1) \bmod n=0, \text { and } \frac{y_{\frac{i+n-1}{n}}}{n}=\tilde{a} ; \\
b_{0}, & \text { if } i \bmod n=0, \text { and } y_{\frac{i}{n}}=\tilde{b} ; \\
b_{1}, & \text { if }(i+1) \bmod n=0, \text { and } \frac{y_{\frac{i+1}{n}}}{n}=\tilde{b} ; \\
\vdots & \vdots \\
b_{n}, & \text { if }(i+n-1) \bmod n=0, \text { and } \frac{y_{\frac{i+n-1}{n}}}{n}=\tilde{b} .
\end{array}\right.
$$

Proposition 2. [19] Releasing transformation $R$ is a continuous and injective map.

Let $\alpha_{i}=\underbrace{(0,1,0,1, \cdots, 0,1,0,1)}_{2 i}, \quad \beta_{i}=\underbrace{(0,0,0,0, \cdots, 0,0,0,0)}_{2 i}, i=1,2,3, \cdots$, and $\tilde{S}_{i}=\left\{\alpha_{i}, \beta_{i}\right\}$ be a new symbolic set. Denote by $\tilde{\Lambda}_{i}$ the subshift in $\tilde{S}^{z}$ determined by the transition matrix as $\tilde{A}=\left(\begin{array}{ll}1 & 1 \\ 1 & 0\end{array}\right)$. Then induce $\tilde{\sigma}: \tilde{\Lambda}_{i} \rightarrow \tilde{\Lambda}_{i}$, where $\tilde{\sigma}$ is the classical left-shift map. And let $\Lambda_{i}$ be $\Lambda_{i}=R\left(\tilde{\Lambda}_{i}\right)$, then induce $\sigma: \Lambda_{i} \rightarrow \Lambda_{i}$, where $i=1,2,3, \cdots$. Then considering $\Lambda_{i} \subseteq \Lambda$ and Proposition 1 , one can easily obtain the following proposition.

Proposition 3. For each $i \in Z^{+}, \Lambda_{i}$ is closed and $T_{106}$-invariant.

Proposition 4. For each $i \in Z^{+},\left(\tilde{\Lambda}_{i}, \tilde{\sigma}\right)$ and $\left(\Lambda_{i}, \sigma^{2 i}\right)$ are topologically conjugate.

Proof: It is clear that the following diagram is commutative. The rest of proof can be completed by applying Proposition 2.

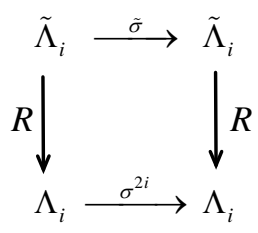

Theorem 3. For each $i \in Z^{+}$, 1) $T_{106}^{2 i}$ is topologically mixing on each $\Lambda_{i}$;

2) the topologically entropy of $T_{106}^{2 i}$ on $\Lambda_{i}$ equals to $\log \frac{1+\sqrt{5}}{2}$; therefore, the topologically entropy of 
$T_{106}$ on $\Lambda_{i}$ equals to $\frac{1}{2 i} \log \frac{1+\sqrt{5}}{2}$.

Proof: 1) It is clear to check that $\tilde{A}$ is irreducible and aperiodic, thus $\tilde{\sigma}$ is topologically mixing on $\tilde{\Lambda}_{i}$, $i=1,2,3, \cdots$. For each $i \in Z,\left(\tilde{\Lambda}_{i}, \tilde{\sigma}\right)$ and $\left(\Lambda_{i}, \sigma^{2 i}\right)$ are topologically conjugate, so $\sigma^{2 i}$ is topologically mixing on each $\Lambda_{i}$ and thus $T_{106}^{2 i}$ is topologically mixing on each $\Lambda_{i}$ based on Proposition 1 and Proposition 3.

2) Since $\operatorname{ent}\left(\left.\tilde{\sigma}\right|_{\Lambda_{i}}\right)=\log (\rho(\tilde{A}))=\log \frac{1+\sqrt{5}}{2}$, where $\rho(\tilde{A})$ is the the spectral radius of the transition matrix $\tilde{A}, \quad i=1,2,3, \cdots$. Therefore, $\operatorname{ent}\left(\left.T_{106}^{2 i}\right|_{\Lambda_{i}}\right)=\operatorname{ent}\left(\left.\tilde{\sigma}\right|_{\tilde{\Lambda}_{i}}\right)=\log \frac{1+\sqrt{5}}{2}$ according to Proposition 3 . Then the topologically entropy of $T_{106}^{2 i}$ on each $\Lambda_{i}$ equals to $\log \frac{1+\sqrt{5}}{2}$; therefore, the topologically entropy of $T_{106}$ on each $\Lambda_{i}$ equals to $\frac{1}{2 i} \log \frac{1+\sqrt{5}}{2}$.

Theorem 4. For each $i \in Z^{+}$, 1) $T_{106}$ is topologically mixing on $\Lambda_{i}$;

2) $T_{106}$ is chaotic in the sense of both Li-Yorke and Devaney on $\Lambda_{i}$.

Proof: 1) One can use the definition of the topologically mixing to prove that $T_{106}$ is topologically mixing on each $\Lambda_{i}$. i.e. for any two nonempty open subsets $U, V \subseteq \Lambda_{i}, \exists N>0, \forall n \geq N$, such that $T_{106}^{n}(U) \cap V \neq \varnothing$. For each $i \in Z^{+}$, the following are two conditions to illustrate:

Case 1. $n=2 i k$. According to theorem $3(1), T_{106}^{2 i}$ is topologically mixing, namely, for any two nonempty open sets $U, V \subseteq \Lambda_{i}, \exists N>0, \forall k \geq N$, such that $\left(T_{106}^{2 i}\right)^{k}(U) \cap V \neq \varnothing$, so $\forall n=2 k>N, T_{106}^{n}(U) \cap V \neq \varnothing$.

Case 2. $n=2 i k+j, \quad j=1,2, \cdots, 2 i k+2 i-1$. Firstly one need to prove that $T_{106}: \Lambda_{i} \rightarrow \Lambda_{i}$ is a homeomorphism. Since $\Lambda_{i} \subseteq \Lambda$ and $\Lambda_{i}$ is $T_{106}$-invariant, then $T_{106}$ is surjective. Suppose that there exist $x_{1}, x_{2} \in \Lambda_{i}$, such that $T_{106}\left(x_{1}\right)=T_{106}\left(x_{2}\right)$, so $T_{106}^{2 i}\left(x_{1}\right)=T_{106}^{2 i}\left(x_{2}\right)$, which implies $\sigma^{2 i}\left(x_{1}\right)=\sigma^{2 i}\left(x_{2}\right)$, thus $x_{1}=x_{2}$. So $T_{106}$ is injective. Since $\Lambda_{i}$ is a compact Hausdorff space, $\left.T_{106}\right|_{\Lambda_{i}}$ is one to one, onto and continuous map. $T_{106}^{-1}: \Lambda_{i} \rightarrow \Lambda_{i}$ exists and continuous. Therefore, $T_{106}: \Lambda_{i} \rightarrow \Lambda_{i}$ is a homeomorphism. This implies that $T_{106}^{j}(U)$ is also an open set, thus, one has $T_{106}^{n}(U) \cap V=T_{106}^{2 i k+j}(U) \cap V=\left(T_{106}^{2 i}\right)^{k}\left(T_{106}^{j}(U)\right) \cap V \neq \varnothing$, where $j=1,2, \cdots, 2 i k+2 i-1$.

2) It is easily deduced by Theorem 3 (2) and Theorem 4 (1).

Note that $\bigcap_{i=1}^{+\infty} \Lambda_{i}=\left\{\alpha_{0}, \beta_{0}\right\}$, where $\alpha_{0}=(\cdots, 0,1, \stackrel{*}{0}, 1,0,1, \cdots), \beta_{0}=(\cdots, 0,1,0,1,0,1, \cdots)$. Let $\Lambda_{i}^{\prime}=\Lambda_{i} \backslash\left\{\alpha_{0}, \beta_{0}\right\}$, then $\bigcap_{i=1}^{\infty} \Lambda_{i}=\varnothing$. Observe that $\sigma\left(\alpha_{0}\right)=\beta_{0}, \sigma\left(\beta_{0}\right)=\alpha_{0}$, then for each $i \in Z^{+}, \Lambda_{i}^{\prime}$ is closed and $\sigma$-invariant. Thus Theorem 3 and 4 also hold for $\Lambda_{i}^{\prime}$, where $i \in Z^{+}$.

Remark 1. It is important to point out that the topologically entropy of $T_{106}$ on $\Lambda_{i}^{\prime}$ approaches 0 as $i$ approaches $+\infty$. Meanwhile, it has been proved that there exists a "big" subsystem of rule 106, including infinite disjoint chaotic subsystems $\Lambda_{1}^{\prime}, \Lambda_{2}^{\prime}, \cdots$. This analytical assertion provides an enlightening fact that the hyper Bernoulli-shift rule 106 is full of infinite "small" chaotic subsystems in a "big" subsystem, demonstrating its very rich and complex dynamics.

\section{Conclusion}

One of the main challenges is to explore the quantitative dynamics in cellular automata evolution. Hyper 
Bernoulli-shift rules possess very interesting and complicated dynamical behaviors [19] [20], for example, rule 180 possesses infinitely many generalized sub-shifts [20] [21]. This paper is devoted to an in-depth study of cellular automaton rule 106 in the framework of symbolic dynamics. Indeed, rule 106 actually is topologically mixing and possesses positive topological entropy on a subsystem. Furthermore, in this chaotic subsystem, rule 106 defines infinitely number of chaotic subsystems with rich and complex dynamical behaviors, such as topologically mixing, positive topological entropies and chaos in the sense of Li-Yorke and Devaney. Although in this work, one obtains some interesting results, to rule 106, it still needs much deeper research in the future.

\section{Acknowledgements}

This research was supported by the NSFC (Grant No. 11171084).

\section{References}

[1] von Neumann, J. and Burks, A.W. (1966) Theory of Self-Reproducing Automata. University of Illinois Press, Urbana and London.

[2] Hedlund, G.A. (1969) Endomorphisms and Automorphism of the Shift Dynamical System. Theory of Computing Systems, 3, 320-375.

[3] Gardner, M. (1970) The Fantastic Combinations of John Conway’s New Solitaire Game Life. Scientific American, 223, 120-123. http://dx.doi.org/10.1038/scientificamerican1070-120

[4] Wolfram, S. (1984) Universality and Complexity in Cellular Automata. Physica D, 10, 1-35. http://dx.doi.org/10.1016/0167-2789(84)90245-8

[5] Wolfram, S. (1986) Theory and Application of Cellular Automata. Word Scientific, Singapore.

[6] Wolfram, S. (2002) A New Kind of Science. Wolfram Media, Inc., Champaign.

[7] Chua, L.O., Sbitnev, V.I. and Yoon, S. (2004) A Nonlinear Dynamics Perspective of Wolfram's New Kind of Science, Part III: Predicting the Unpredictable. International Journal of Bifurcation and Chaos, 14, 3689-3820. http://dx.doi.org/10.1142/S0218127404011764

[8] Chua, L.O., Guan, J., Sbitnev, V.I. and Jinwook, S. (2007) A Nonlinear Dynamics Perspective of Wolfram's New Kind of Science, Part VII: Isles of Eden. International Journal of Bifurcation and Chaos, 17, 2839-3012. http://dx.doi.org/10.1142/S0218127407019068

[9] Chua, L.O., Karacs, K., Sbitnev, V.I., Guan, J. and Jinwook, S. (2007) A Nonlinear Dynamics Perspective of Wolfram's New Kind of Science, Part VIII: More Isles of Eden. International Journal of Bifurcation and Chaos, 17, 3741-3894. http://dx.doi.org/10.1142/S0218127407019901

[10] Chua, L.O. and Pazienza, G.E. (2010) A Nonlinear Dynamics Perspective of Wolfram's New Kind of Science, Part XIV: More Bernoulli $\sigma_{\tau}$-Shift Rules. International Journal of Bifurcation and Chaos, 20, 2253-2425. http://dx.doi.org/10.1142/S0218127410027155

[11] Guan, J.B., Shen, S.W., Tang, C.B. and Chen, F.Y. (2007) Extending Chua's Global Equivalence Theorem on Wolfram's New Kind of Science. International Journal of Bifurcation and Chaos, 17, 4245-4259. http://dx.doi.org/10.1142/S0218127407019925

[12] Chen, F.Y., Jin, W.F., Chen, G.R., Chen, F.F. and Chen, L. (2009) Chaos of Elementary Cellular Automata Rule 42 of Wolfram's Class II. Chaos, 19, Article ID: 013140.

[13] Chen, F.Y., Chen, G. and Jin, W.F. (2013) Transitivity and Chaoticity in 1-D Cellular Automata. International Journal of Modern Nonlinear Theory and Application, 2, 69-73. http://dx.doi.org/10.4236/ijmnta.2013.21A008

[14] Jin, W.F., Chen, F.Y., Chen, G.R., Chen, L. and Chen, F.F. (2010) Extending the Symbolic Dynamics of Chua’s Bernoulli-Shift Rule 56. Journal of Cellular Automata, 5, 121-138.

[15] Chen, G.R., Chen, F.Y., Guan, J.B. and Jin, W.F. (2010) Symbolic Dynamics of Some Bernoullif-Shift Cellular Automata Rules. 2010 International Symposium on Nonlinear Theory and Its Applications, 595-598.

[16] Cattaneo, G., Finelli, M. and Margara, L. (2000) Investigating Topological Chaos by Elementary Cellular Automata Dynamics. Theoretical Computer Science, 244, 219-241. http://dx.doi.org/10.1016/S0304-3975(98)00345-4

[17] Kitchens, B. (1998) Symbolic Dynamics: One-Sided, Two-Sided and Countable State Markov Shifts. Springer-Verlag, Berlin. http://dx.doi.org/10.1007/978-3-642-58822-8

[18] Blanchard, F., Glasner, E. and Kolyada, S. (2002) On Li-Yorke Pairs. Journal fur reine und angewandte Mathematik, 547, 51-68. 
[19] Jin, W.F. and Chen, F.Y. (2011) Topological Chaos of Universal Elementary Cellular Automata Rule. Nonlinear Dynamics, 63, 217-222. http://dx.doi.org/10.1007/s11071-010-9798-z

[20] Chen, W., Chen, F.Y., Bian, Y.F. and Chen, J. (2011) Infinite Number of Chaotic Generalized Sub-Shifts of Cellular Automaton Rule 180. 2011 International Conference on Scientific Computing, 226-230.

[21] Cattaneo, G. and Margara, L. (1998) Generalized Sub-Shifts in Elementary Cellular Automata: The "Strange Case” of Chaotic Rule 180. Theoretical Computer Science, 201, 171-187. http://dx.doi.org/10.1016/S0304-3975(97)00210-7 
Scientific Research Publishing (SCIRP) is one of the largest Open Access journal publishers. It is currently publishing more than 200 open access, online, peer-reviewed journals covering a wide range of academic disciplines. SCIRP serves the worldwide academic communities and contributes to the progress and application of science with its publication.

Other selected journals from SCIRP are listed as below. Submit your manuscript to us via either submit@scirp.org or Online Submission Portal.
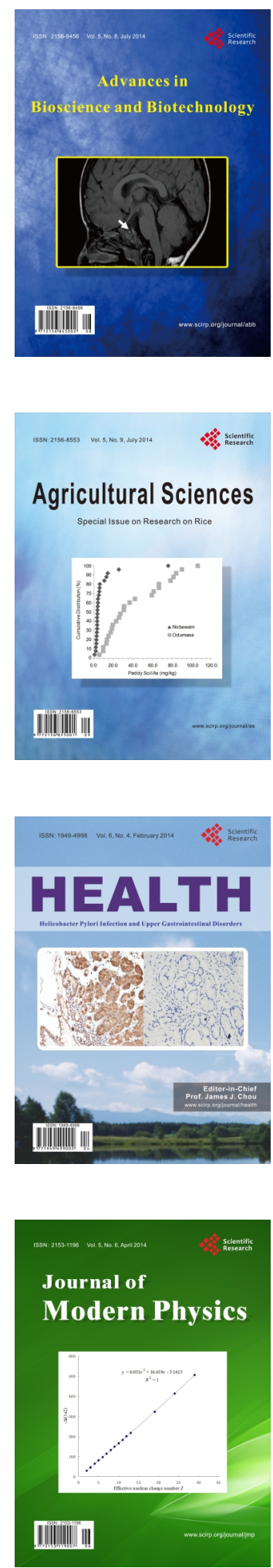
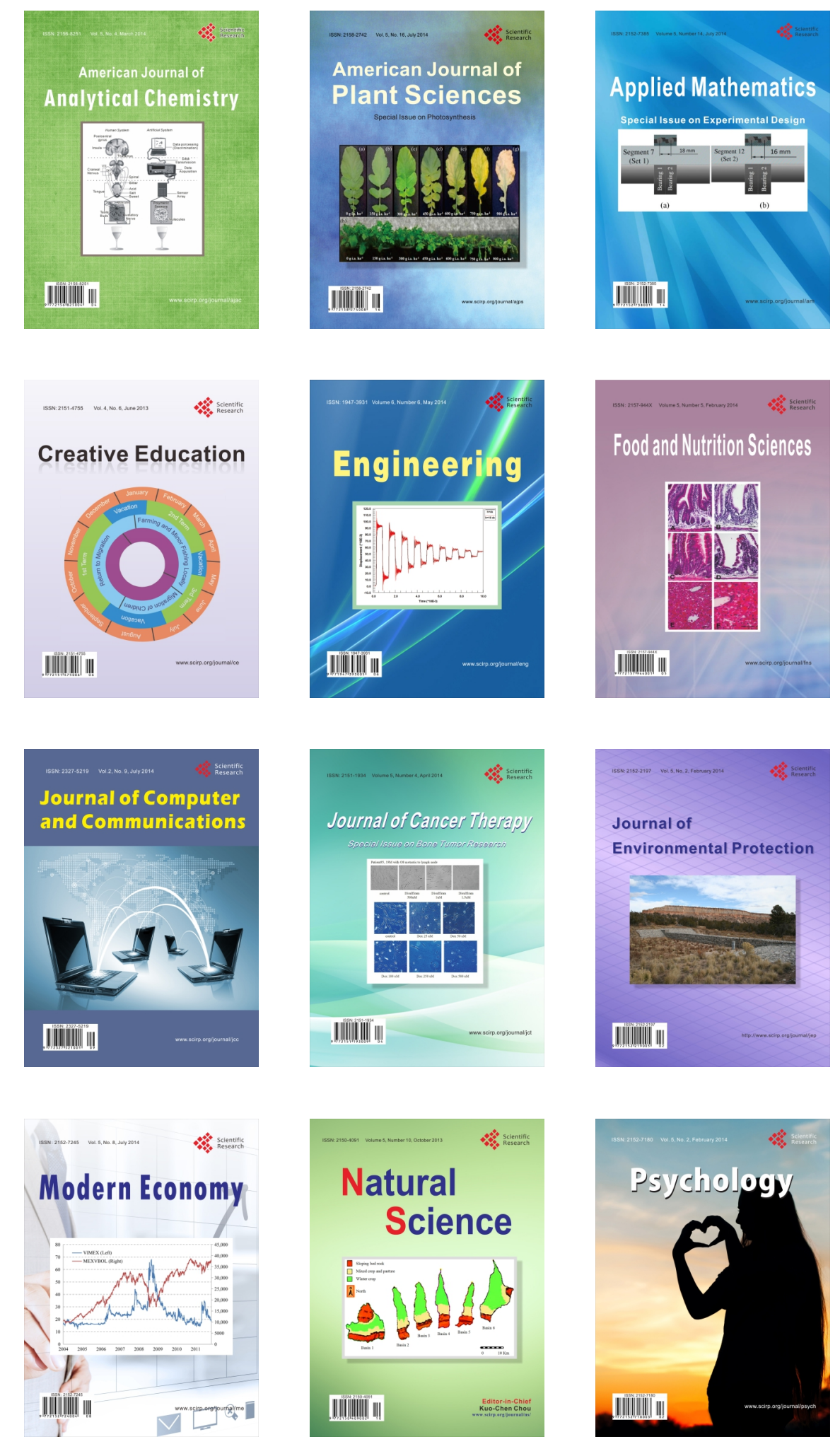